TITLE:

\title{
Electronic structures of dynamically stable As2O3, Sb2O3, and Bi2O3 crystal polymorphs
}

\section{$\operatorname{AUTHOR}(S)$ :}

Matsumoto, Akifumi; Koyama, Yukinori; Togo, Atsushi; Choi, Minseok; Tanaka, Isao

\section{CITATION:}

Matsumoto, Akifumi ...[et al]. Electronic structures of dynamically stable As203, Sb2O3, and Bi2O3 crystal polymorphs. PHYSICAL REVIEW B 2011, 83(21): 214110.

ISSUE DATE:

2011-06

URL:

http://hdl.handle.net/2433/161785

RIGHT:

(C2011 American Physical Society 
PHYSICAL REVIEW B 83, 214110 (2011)

\title{
Electronic structures of dynamically stable $\mathrm{As}_{2} \mathrm{O}_{3}, \mathrm{Sb}_{2} \mathrm{O}_{3}$, and $\mathrm{Bi}_{2} \mathrm{O}_{3}$ crystal polymorphs
}

\author{
Akifumi Matsumoto, ${ }^{1}$ Yukinori Koyama, ${ }^{1}$ Atsushi Togo, ${ }^{1}$ Minseok Choi, ${ }^{1}$ and Isao Tanaka ${ }^{1,2,3, *}$ \\ ${ }^{1}$ Department of Materials Science and Engineering, Kyoto University, Kyoto 606-8501, Japan \\ ${ }^{2}$ Nanostructures Research Laboratory, Japan Fine Ceramics Center, Nagoya 456-8587, Japan \\ ${ }^{3}$ Fukui Institute for Fundamental Chemistry, Kyoto University, Kyoto 606-8103, Japan \\ (Received 7 February 2011; revised manuscript received 13 March 2011; published 17 June 2011)
}

\begin{abstract}
The relationships between the atomic arrangements, electronic structures, and energetics of three sesquioxides, $\mathrm{As}_{2} \mathrm{O}_{3}, \mathrm{Sb}_{2} \mathrm{O}_{3}$, and $\mathrm{Bi}_{2} \mathrm{O}_{3}$, are systematically investigated by first-principles lattice-dynamics calculations. Dynamically stable structures of the defective fluorite family are searched for by including atomic displacements along imaginary modes of lattice vibrations that appear in high-symmetry structures. Experimentally reported crystal structures (arsenolite- $\mathrm{As}_{2} \mathrm{O}_{3}, \alpha-\mathrm{Sb}_{2} \mathrm{O}_{3}, \beta-\mathrm{Sb}_{2} \mathrm{O}_{3}$, and $\beta-\mathrm{Bi}_{2} \mathrm{O}_{3}$ ) are found to be formed by symmetry breaking and local atomic distortion in this way. Moreover, some dynamically stable structures that have not yet been revealed by experiments are discovered. Their electronic structures and the magnitudes of their band gaps are obtained. In the dynamically stable low-symmetry structures, valence electrons localize and form an asymmetric charge distribution along the direction of an empty anion site of the defective fluorite structure. This is a common characteristic of a "lone pair" of cations. The formation of the lone pair is less significant in the order $\mathrm{As}_{2} \mathrm{O}_{3}, \mathrm{Sb}_{2} \mathrm{O}_{3}, \mathrm{Bi}_{2} \mathrm{O}_{3}$, which is the same trend as the lattice distortion. The formation of the lone pair plays an essential role in determining the structures, stability, and properties of these sesquioxides.
\end{abstract}

DOI: 10.1103/PhysRevB.83.214110

PACS number(s): 61.50.Ah, 63.20.-e, 71.20.-b

\section{INTRODUCTION}

Crystal structures of simple metal oxides are often composed of a close-packed oxide-ion sublattice with its tetrahedral or octahedral interstitial sites occupied by cations. Sesquioxides of late group-15 elements in the Periodic Table, i.e., $\mathrm{As}_{2} \mathrm{O}_{3}, \mathrm{Sb}_{2} \mathrm{O}_{3}$, and $\mathrm{Bi}_{2} \mathrm{O}_{3}$, form several polymorph structures that are far from such simple metal oxides. They form highly distorted structures with large open spaces (or low atomic density). It is generally accepted that the atomic distortion can be ascribed to the presence of "lone pair" electrons, because two $n s$ ( $n=4,5$, and 6) electrons remain in $\mathrm{As}^{3+}, \mathrm{Sb}^{3+}$, and $\mathrm{Bi}^{3+}$ in the formal sense, respectively. However, the relationships between their crystal structures, electronic structures, and energetics have not been quantitatively investigated.

It has been experimentally shown that $\mathrm{As}_{2} \mathrm{O}_{3}$ exhibits two polymorphs: cubic arsenolite and monoclinic claudetite. ${ }^{1-4}$ $\mathrm{Sb}_{2} \mathrm{O}_{3}$ is also known to exhibit two polymorphs: cubic $\alpha$-phase (senarmontite) and orthorhombic $\beta$-phase (valentinite) ${ }^{5-9}$ $\mathrm{Bi}_{2} \mathrm{O}_{3}$ has been reported to form six polymorphs: monoclinic $\alpha$-phase, tetragonal $\beta$-phase, cubic $\gamma$-phase, cubic $\delta$-phase, orthorhombic $\varepsilon$-phase, and triclinic $\omega$-phase. ${ }^{10-20}$ The structures of these three sesquioxides are closely related. The structure of arsenolite- $\mathrm{As}_{2} \mathrm{O}_{3}$ is the same as that of $\alpha-\mathrm{Sb}_{2} \mathrm{O}_{3} \cdot \beta-\mathrm{Sb}_{2} \mathrm{O}_{3}$ is isostructural to $\varepsilon-\mathrm{Bi}_{2} \mathrm{O}_{3}$.

The physical properties of crystals are generally strongly dependent on their structures. For example, $\mathrm{Bi}_{2} \mathrm{O}_{3}$ exhibits high oxide ionic conductivity in the $\delta$ phase while the other phases show very low ionic conductivity. ${ }^{21}$ Understanding the property-structure relationships is therefore essential for the selection of materials. Although these three sesquioxides have similarities in their structures, systematic studies on them have not been reported. In this study, the energetics and crystal and/or electronic structures of $M_{2} \mathrm{O}_{3}(M=\mathrm{As}, \mathrm{Sb}, \mathrm{Bi})$ polymorphs are systematically investigated by first-principles calculations.
The structures of these sesquioxides appear to be quite diverse. However, a major part of them can be included in a defective fluorite family, which is based on a fluorite structure in which a quarter of the $\mathrm{O}$ sites are vacant. The term "array" will be used hereafter to refer to the atomic arrangement in the O sites of the fluorite structure, while the term "arrangement" will retain its general meaning and refer to a set of specific atomic positions. Arsenolite- $\mathrm{As}_{2} \mathrm{O}_{3}$ and $\alpha-\mathrm{Sb}_{2} \mathrm{O}_{3}$ are classified into the $\langle 111\rangle$ array model, in which the vacant sites are aligned in the $\langle 111\rangle$ direction. $\beta-\mathrm{Bi}_{2} \mathrm{O}_{3}$ is classified into the $\langle 100\rangle$ array model, in which the vacant sites are aligned in the $\langle 100\rangle$ direction. $\beta-\mathrm{Sb}_{2} \mathrm{O}_{3}$ and $\varepsilon-\mathrm{Bi}_{2} \mathrm{O}_{3}$ are classified into the valentinite-type array model, which is made by doubling the defective fluorite unit cell with the [100] array in the [001] direction along with the translation of the vacancy array by half the unit-cell size in the [010] direction. In our previous report, ${ }^{22}$ the structures of defective fluorite $\mathrm{Bi}_{2} \mathrm{O}_{3}$ family members were investigated in detail. A series of dynamically stable structures was found by lowering the symmetry along imaginary modes of lattice vibrations from the high-symmetry defective fluorite structure. The experimentally reported structure of $\beta-\mathrm{Bi}_{2} \mathrm{O}_{3}{ }^{20}$ was obtained from the high-symmetry $\langle 100\rangle$ array model in this way. It was also emphasized that the clear band gap of $\mathrm{Bi}_{2} \mathrm{O}_{3}$ can be found only when such symmetry breaking is included for the $\langle 100\rangle,\langle 110\rangle$, and $\langle 111\rangle$ array models. In the present study, we search for dynamically stable structures of $\mathrm{As}_{2} \mathrm{O}_{3}$ and $\mathrm{Sb}_{2} \mathrm{O}_{3}$ in the same manner as we did for those of $\mathrm{Bi}_{2} \mathrm{O}_{3}$, as described in our previous report. ${ }^{22}$ Then the electronic origin of their structures and energetics is discussed.

\section{COMPUTATIONAL PROCEDURES}

Five array models based on the defective fluorite structure $(\langle 100\rangle,\langle 110\rangle,\langle 111\rangle$, bixbyite-type, and valentinite-type array models) were examined for the three sesquioxides. The five 

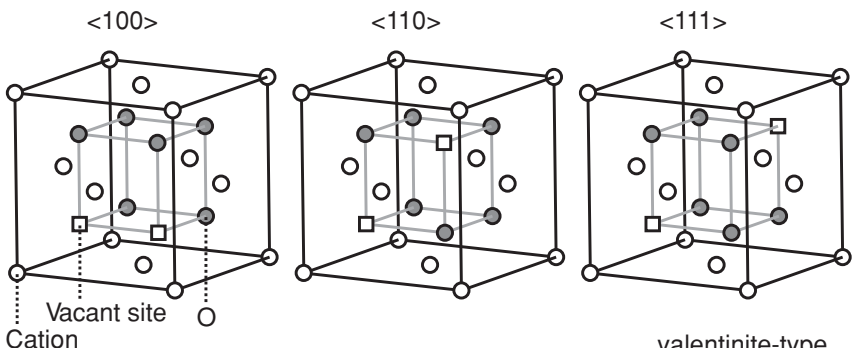

Cation

bixbyite-type
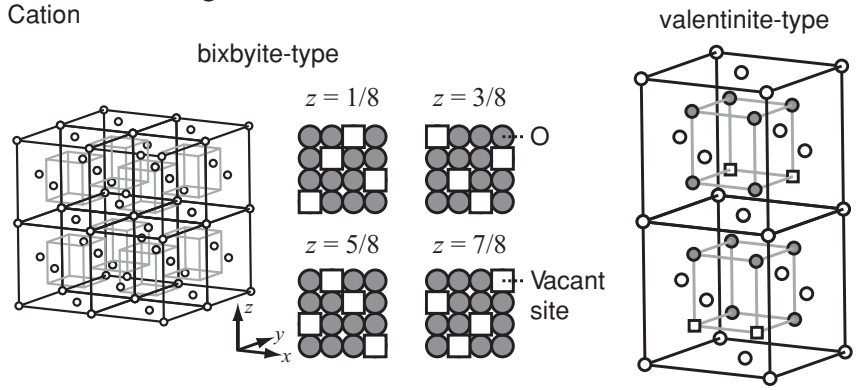

FIG. 1. The five array models of the defective fluorite structure. Since the bixbyite-type array is complicated, the array in each layer is drawn to the right.

array models of the defective fluorite structure are shown in Fig. 1. Bixbyite- and valentinite-type array models can be made in the $2 \times 2 \times 2$ and $1 \times 1 \times 2$ supercells of the defective fluorite unit cell, respectively. For comparison, two different kinds of atomic arrangements within the monoclinic space group of $P 2_{1} / c$ were examined, which were taken from experimentally reported structures of claudetite- $\mathrm{As}_{2} \mathrm{O}_{3}$ (Ref. 23) and $\alpha$ $\mathrm{Bi}_{2} \mathrm{O}_{3} \cdot{ }^{24}$

The first-principles calculations were performed by the projector augmented wave (PAW) method ${ }^{25}$ implemented in the VASP code. ${ }^{26-28}$ The exchange-correlation term was treated with the Perdew-Burke-Ernzerhof (PBE) functional based on the generalized gradient approximation (GGA) ${ }^{29}$ Plane waves were used as the basis functions with a cutoff energy of $360 \mathrm{eV}$. The convergence of the formation energy with respect to the plane-wave cutoff was found to be better than $0.01 \mathrm{eV} /$ formula unit. The radii of the PAW potentials were $1.11,1.21,1.32$, and $0.98 \AA$ for $\mathrm{As}, \mathrm{Sb}, \mathrm{Bi}$, and $\mathrm{O}$, respectively. The $4 s$ and $4 p$ electrons for As; $5 s$ and $5 p$ electrons for $\mathrm{Sb}$; $5 d, 6 s$, and $6 p$ electrons for $\mathrm{Bi}$; and $2 s$ and $2 p$ electrons for $\mathrm{O}$ were treated as valence and the remaining electrons were kept frozen. In the calculation of the charge density in Sec. III D, the $5 d$ electrons of $\mathrm{Bi}$ were kept frozen to match the number of valence electrons with that of As and Sb. Integration in the reciprocal space was performed by the Monkhorst-Pack scheme $^{30}$ using the $4 \times 4 \times 4$ mesh for the $\langle 100\rangle,\langle 110\rangle$, and $\langle 111\rangle$ array models, the $4 \times 4 \times 2$ mesh for the valentinite-type array model, the $2 \times 2 \times 2$ mesh for the bixbyite-type array model, the $3 \times 6 \times 2$ mesh for the claudetite- $\mathrm{As}_{2} \mathrm{O}_{3}$ model, and the $3 \times 2 \times 2$ mesh for the $\alpha-\mathrm{Bi}_{2} \mathrm{O}_{3}$ model. The total energy was minimized until the energy convergence became less than $1 \times 10^{-5} \mathrm{eV}$.

To examine the stability of the model structures against lattice dynamics, the force-constant approach involving a finite displacement was used employing the FROPHO code. ${ }^{31-33}$ The $2 \times 2 \times 2$ supercells of the defective fluorite structure were used with a displacement of $0.01 \AA$ along the $x, y$, and $z$ directions for each atom.

\section{RESULTS AND DISCUSSION}

\section{A. Symmetry breaking from ideal defective fluorite structures}

First a set of defective fluorite structures with the five different array models was optimized by imposing the highest symmetry that can accommodate the array of vacant sites in each defective fluorite structure. Then the $\langle 111\rangle$ and bixbyite-type array models formed a cubic lattice, the $\langle 100\rangle$ and $\langle 110\rangle$ array models formed a tetragonal lattice, and the valentinite-type array model formed an orthorhombic lattice. These will be referred to hereafter as high-symmetry (HS) structures. Lattice-dynamics calculations were performed for these structures. Examples of phonon band structures for the $\langle 111\rangle$ array models of the three sesquioxides are shown in Fig. 2. An imaginary value of the frequency illustrated below 0 indicates that the structure is at a local maximum of the potential surface and is unstable against the corresponding lattice vibration. All the HS defective fluorite structures of the three sesquioxides with the five different arrays show such imaginary frequencies and thus are dynamically unstable.

These HS structures were distorted by adding atomic displacements of less than $0.5 \AA$ according to the vibration mode with the largest absolute value of the imaginary frequency. This
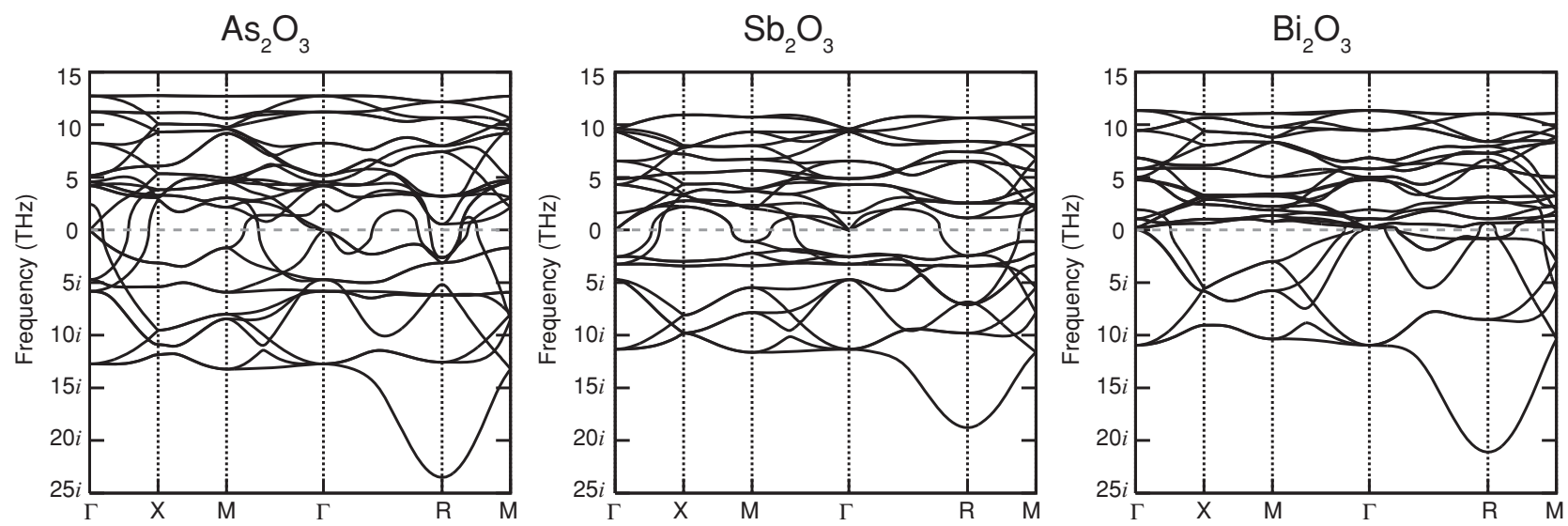

FIG. 2. Phonon band structures of the $\langle 111\rangle$-HS arrangements of the three sesquioxides. 
TABLE I. Details of calculated LS structures compared with experimentally reported structures of $\mathrm{As}_{2} \mathrm{O}_{3}$.

\begin{tabular}{|c|c|c|c|c|c|c|c|c|c|c|c|}
\hline & \multicolumn{5}{|c|}{$<100>-\mathrm{LS}-\mathrm{As}_{2} \mathrm{O}_{3}$} & & \multicolumn{5}{|c|}{ valentinite-LS- $\mathrm{As}_{2} \mathrm{O}_{3}$} \\
\hline Space Group & \multicolumn{5}{|c|}{$\mathrm{P}_{2} / n \quad$ (No. 86) } & Space Group & \multicolumn{5}{|c|}{$P c c 2 \quad$ (No. 27) } \\
\hline \multirow{2}{*}{ Lattice Parameter } & \multicolumn{5}{|c|}{$a=7.04 \AA$} & \multirow{3}{*}{ Lattice Parameter } & \multirow{2}{*}{\multicolumn{5}{|c|}{$\begin{array}{c}a=12.57 \AA \\
b=5.99 \AA\end{array}$}} \\
\hline & & & $c=8.3$ & & & & & & & & \\
\hline \multirow{7}{*}{ Atom Coordinate } & As (1) & $8 g$ & 0.047 & 0.274 & 0.604 & & & & $c=5.25$ & & \\
\hline & $\mathrm{O}(1)$ & $8 g$ & 0.158 & 0.183 & 0.420 & \multirow{6}{*}{ Atom Coordinate } & As (1) & $4 e$ & 0.120 & 0.357 & 0.494 \\
\hline & $\mathrm{O}(2)$ & $4 e$ & 0 & 0.5 & 0.002 & & As (2) & $4 e$ & 0.380 & 0.143 & 0.420 \\
\hline & & & & & & & $\mathrm{O}(1)$ & $2 b$ & 0 & 0.5 & 0.115 \\
\hline & & & & & & & $\mathrm{O}(2)$ & $2 c$ & 0.5 & 0 & 0.114 \\
\hline & & & & & & & $\mathrm{O}(3)$ & $4 e$ & 0.316 & 0.081 & 0.301 \\
\hline & & & & & & & $\mathrm{O}(4)$ & $4 e$ & 0.184 & 0.581 & 0.301 \\
\hline
\end{tabular}

\begin{tabular}{|c|c|c|c|c|c|c|c|c|c|c|}
\hline & \multicolumn{5}{|c|}{$<111>-$ LS- $\mathrm{A}_{2} \mathrm{O}_{3}$} & \multicolumn{5}{|c|}{ arsenolite (experimental data ${ }^{1}$ ) } \\
\hline Space Group & \multicolumn{5}{|c|}{$F d \overline{3} m \quad$ (No. 227) } & \multicolumn{5}{|c|}{$F d \overline{3} m \quad$ (No. 227) } \\
\hline Lattice Parameter & \multicolumn{5}{|c|}{$a=11.54 \AA$} & \multicolumn{5}{|c|}{$a=11.07 \AA$} \\
\hline \multirow{2}{*}{ Atom Coordinate } & As (1) & $32 e$ & 0.899 & 0.899 & 0.899 & As (1) & $32 e$ & 0.897 & 0.897 & 0.897 \\
\hline & $\mathrm{O}(1)$ & $48 f$ & 0.172 & 0 & 0 & $\mathrm{O}(1)$ & $48 f$ & 0.173 & 0 & 0 \\
\hline
\end{tabular}

\begin{tabular}{|c|c|c|c|c|c|c|c|c|c|c|}
\hline & \multicolumn{5}{|c|}{ claudetite- $\mathrm{As}_{2} \mathrm{O}_{3}$} & \multicolumn{5}{|c|}{ claudetite (experimental data ${ }^{23}$ ) } \\
\hline Space Group & \multicolumn{5}{|c|}{$P 2_{1} / c \quad$ (No. 14) } & \multicolumn{5}{|c|}{$P 2_{1} / c \quad$ (No. 14) } \\
\hline \multirow{4}{*}{ Lattice Parameter } & \multicolumn{5}{|c|}{$a=9.82 \AA$} & \multicolumn{5}{|c|}{$a=9.11 \AA$} \\
\hline & \multicolumn{5}{|c|}{$b=4.69 \AA$} & \multicolumn{5}{|c|}{$b=4.64 \AA$} \\
\hline & \multicolumn{5}{|c|}{$c=14.05 \AA$} & \multicolumn{5}{|c|}{$c=13.28 \AA$} \\
\hline & \multicolumn{5}{|c|}{$\beta=144.47^{\circ}$} & \multicolumn{5}{|c|}{$\beta=143.90^{\circ}$} \\
\hline \multirow{5}{*}{ Atom Coordinate } & As (1) & $4 e$ & 0.681 & 0.835 & 0.382 & As (1) & $4 e$ & 0.685 & 0.831 & 0.383 \\
\hline & As (2) & $4 e$ & 0.182 & 0.299 & 0.818 & As (2) & $4 e$ & 0.188 & 0.292 & 0.816 \\
\hline & $\mathrm{O}(1)$ & $4 e$ & 0.608 & 0.460 & 0.322 & $\mathrm{O}(1)$ & $4 e$ & 0.614 & 0.459 & 0.333 \\
\hline & $\mathrm{O}(2)$ & $4 e$ & 0.955 & 0.149 & 0.769 & $\mathrm{O}(2)$ & $4 e$ & 0.946 & 0.140 & 0.762 \\
\hline & $\mathrm{O}(3)$ & $4 e$ & 0.394 & 0.358 & 0.033 & $\mathrm{O}(3)$ & $4 e$ & 0.401 & 0.349 & 0.034 \\
\hline
\end{tabular}

atomic displacement lowered the symmetry. The structures were then optimized again with the new symmetry.

The lattice-dynamics calculations were performed on the newly obtained structures. If the imaginary mode still remained, the same procedure was repeated until it disappeared at all commensurate points in the reciprocal space of the given cell. Commensurate points correspond to the wave vectors commensurate with the periodicity of the cell under examination. A thus obtained dynamically stable structure showing no imaginary modes at the commensurate points, is hereafter referred to as a low-symmetry (LS) structure. Note that both the new arrangement and the original arrangement are classified into the same array model. However, the symmetry is lower in the new arrangement. If the atomic displacements during the geometric optimization were too large to be described within the same oxygen atomic array model, the procedure was terminated.

The $\langle 111\rangle$-HS arrangement of $\mathrm{As}_{2} \mathrm{O}_{3}$, which is obtained by optimizing the ideal defective fluorite structure with its symmetry, exhibits various imaginary modes as shown in 
TABLE II. Details of calculated LS structures compared with experimentally reported structures of $\mathrm{Sb}_{2} \mathrm{O}_{3}$.

\begin{tabular}{|c|c|c|c|c|c|c|c|c|c|c|}
\hline & \multicolumn{5}{|c|}{$<111>-\mathrm{LS}-\mathrm{Sb}_{2} \mathrm{O}_{3}$} & \multicolumn{5}{|c|}{$\alpha-\mathrm{Sb}_{2} \mathrm{O}_{3}$ (experimental data $\left.{ }^{6}\right)$} \\
\hline Space Group & \multicolumn{5}{|c|}{$F d \overline{3} m$ (No. 227) } & \multicolumn{5}{|c|}{$F d \overline{3} m \quad($ No. 227$)$} \\
\hline Lattice Parameter & \multicolumn{5}{|c|}{$a=11.42 \AA$} & \multicolumn{5}{|c|}{$a=11.15 \AA$} \\
\hline \multirow{2}{*}{ Atom Coordinate } & $\mathrm{Sb}(1)$ & $32 e$ & 0.887 & 0.887 & 0.887 & $\mathrm{Sb}(1)$ & $32 e$ & 0.885 & 0.885 & 0.885 \\
\hline & $\mathrm{O}(1)$ & $48 f$ & 0.186 & 0 & 0 & $\mathrm{O}(1)$ & $48 f$ & 0.186 & 0 & 0 \\
\hline
\end{tabular}

\begin{tabular}{|c|c|c|c|c|c|c|c|c|c|c|}
\hline \multirow[b]{2}{*}{ Space Group } & \multicolumn{5}{|c|}{ valentinite-LS- $\mathrm{Sb}_{2} \mathrm{O}_{3}$} & \multicolumn{5}{|c|}{$\beta-\mathrm{Sb}_{2} \mathrm{O}_{3}\left(\right.$ experimental data $\left.{ }^{6}\right)$} \\
\hline & \multicolumn{5}{|c|}{ Pccn (No. 56) } & \multicolumn{5}{|c|}{ Pccn (No. 56) } \\
\hline \multirow{3}{*}{ Lattice Parameter } & \multicolumn{5}{|c|}{$a=5.20 \AA$} & \multicolumn{5}{|c|}{$a=4.91 \AA$} \\
\hline & \multicolumn{5}{|c|}{$b=12.56 \AA$} & \multicolumn{5}{|c|}{$b=12.46 \AA$} \\
\hline & \multicolumn{5}{|c|}{$c=5.52 \AA$} & \multicolumn{5}{|c|}{$c=5.41 \AA$} \\
\hline \multirow{3}{*}{ Atom Coordinate } & $\mathrm{Sb}(1)$ & $8 e$ & 0.053 & 0.127 & 0.180 & $\mathrm{Sb}(1)$ & $8 e$ & 0.041 & 0.127 & 0.178 \\
\hline & $\mathrm{O}(1)$ & $8 e$ & 0.25 & 0.25 & 0.018 & $\mathrm{O}(1)$ & $8 e$ & 0.25 & 0.25 & 0.023 \\
\hline & $\mathrm{O}(2)$ & $4 c$ & 0.151 & 0.057 & 0.857 & $\mathrm{O}(2)$ & $4 c$ & 0.152 & 0.059 & 0.855 \\
\hline
\end{tabular}

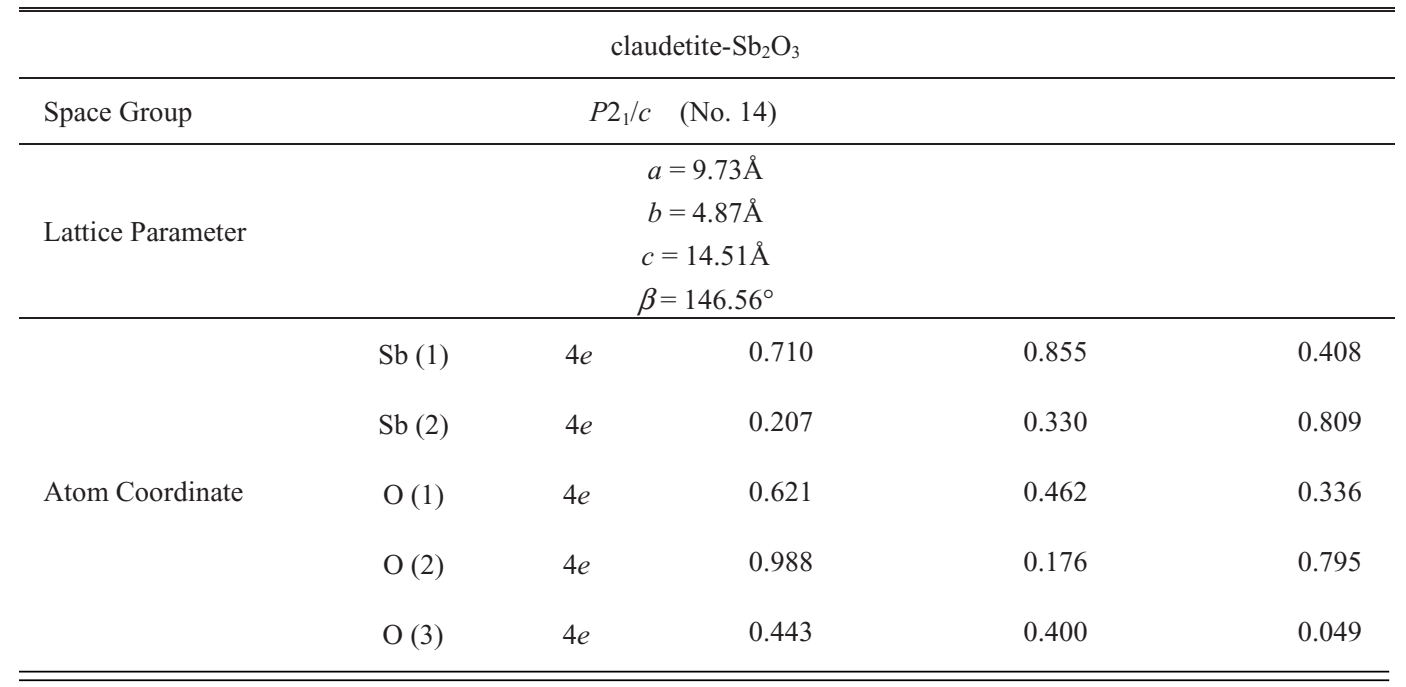

Fig. 2. The displacement along the strongest imaginary mode leads to the $\langle 111\rangle$-LS arrangement. The symmetry and atomic coordinates of the $\langle 111\rangle$-LS arrangement of $\mathrm{As}_{2} \mathrm{O}_{3}$ were found to be in good agreement with the experimentally reported structure of arsenolite- $\mathrm{As}_{2} \mathrm{O}_{3} .{ }^{1}$ In the other array models, dynamically stable $\langle 100\rangle$-LS and valentinite-LS arrangements were found. Table I shows the details of the calculated LS structures and experimentally reported structures of $\mathrm{As}_{2} \mathrm{O}_{3}$.

For $\mathrm{Sb}_{2} \mathrm{O}_{3}$, the $\langle 111\rangle$-HS and valentinite-HS arrangements change to dynamically stable $\langle 111\rangle$-LS and valentinite-LS arrangements, respectively. They are isostructural to the experimentally reported structures of $\alpha-\mathrm{Sb}_{2} \mathrm{O}_{3}$ and $\beta-\mathrm{Sb}_{2} \mathrm{O}_{3} .{ }^{5}$
The structural data of the dynamically stable arrangements and experimentally reported structures are shown in Table II.

In addition to the sets of defective fluorite structures, two different kinds of monoclinic structures with the space group of $P 2_{1} / c$ were examined. The energy of the claudetite- $\mathrm{As}_{2} \mathrm{O}_{3}$ type arrangement is lower than that of the $\alpha-\mathrm{Bi}_{2} \mathrm{O}_{3}$-type arrangement by $0.23 \mathrm{eV} /$ f.u. in $\mathrm{As}_{2} \mathrm{O}_{3}$ and by $0.12 \mathrm{eV} /$ f.u. in $\mathrm{Sb}_{2} \mathrm{O}_{3}$. On the other hand, the $\alpha-\mathrm{Bi}_{2} \mathrm{O}_{3}$-type arrangement is more stable than the claudetite- $\mathrm{As}_{2} \mathrm{O}_{3}$-type arrangement by $0.13 \mathrm{eV} /$ f.u. in $\mathrm{Bi}_{2} \mathrm{O}_{3}$. The energetically lower structure is hereafter referred to as the $P 2_{1} / c$ model of each sesquioxide. These $P 2_{1} / c$ models were found to be dynamically stable. The 


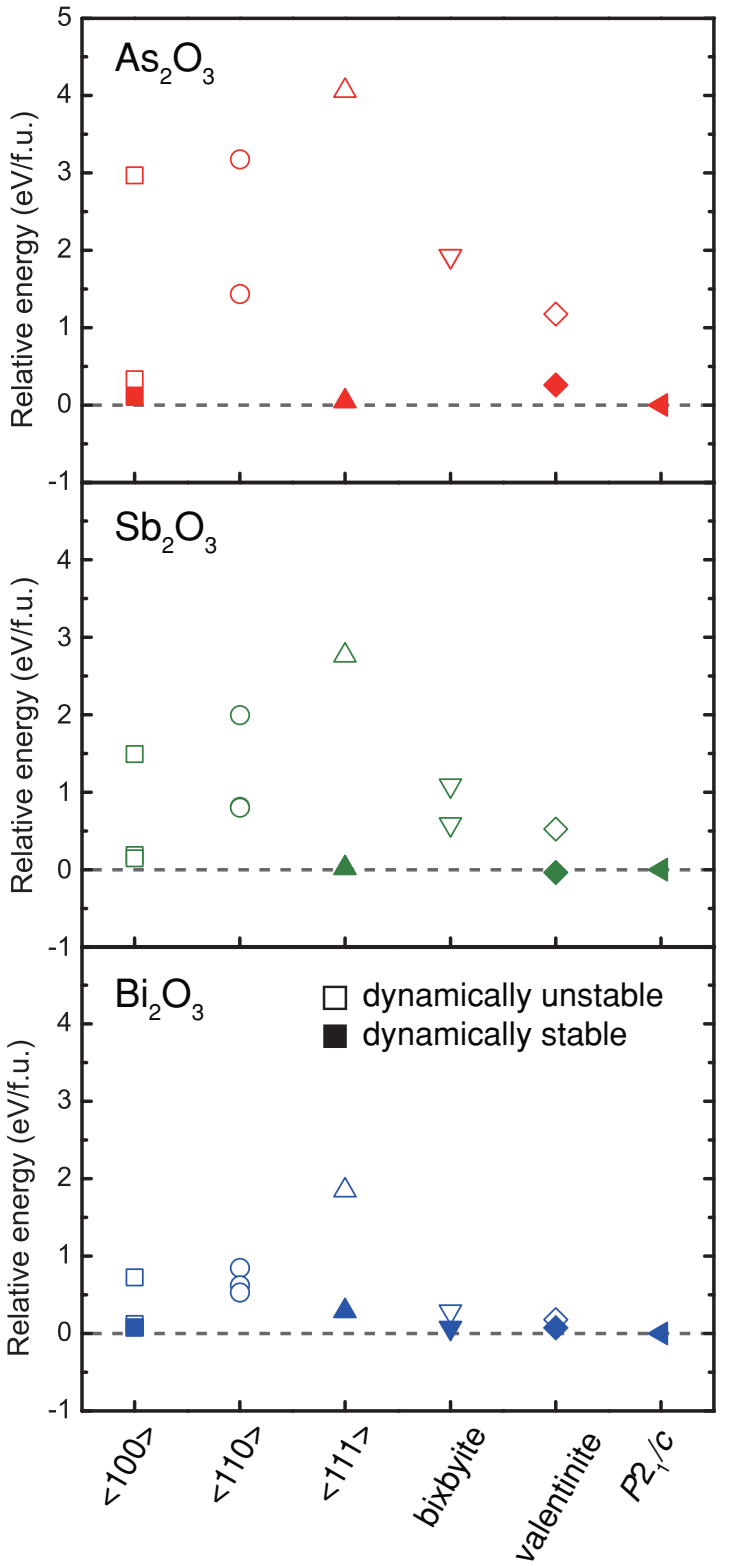

FIG. 3. (Color online) Energies of each arrangement relative to the monoclinic $P 2_{1} / c$ structure.

energies of each arrangement relative to the $P 2_{1} / c$ models are summarized in Fig. 3. The open marks correspond to the dynamically unstable arrangements, and the solid marks correspond to the dynamically stable LS arrangements.

In $\mathrm{As}_{2} \mathrm{O}_{3}$, three of the dynamically stable defective fluorite structures $(\langle 100\rangle$-LS, $\langle 111\rangle$-LS, and valentinite-LS arrangements) exhibit relative energies of less than $0.1 \mathrm{eV} /$ f.u. Although arsenolite- $\mathrm{As}_{2} \mathrm{O}_{3}$ is the low-temperature phase according to an experimental report, ${ }^{34}$ the corresponding arrangement, $\langle 111\rangle$-LS, has $0.05 \mathrm{eV} /$ f.u. higher energy than the claudetite- $\mathrm{As}_{2} \mathrm{O}_{3}$-type arrangement. Similarly to $\mathrm{As}_{2} \mathrm{O}_{3}$, the energetic hierarchy between $\alpha-\mathrm{Sb}_{2} \mathrm{O}_{3}$ and $\beta-\mathrm{Sb}_{2} \mathrm{O}_{3}$ according to the present calculations does not agree with experimental one. The relative energy of the low-temperature phase $\left(\alpha-\mathrm{Sb}_{2} \mathrm{O}_{3}\right)$, which corresponds to the $\langle 111\rangle$-LS arrangement, is $0.06 \mathrm{eV} /$ f.u. higher than that of the high-temperature phase
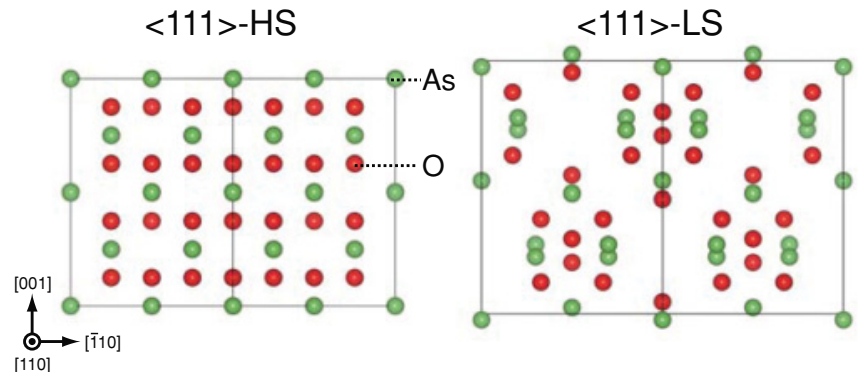

FIG. 4. (Color online) Projection of the $\langle 111\rangle-\mathrm{HS}$ and -LS arrangements of $\mathrm{As}_{2} \mathrm{O}_{3}$ from the [110] direction.

$\left(\beta-\mathrm{Sb}_{2} \mathrm{O}_{3}\right)$, which corresponds to the valentinite-LS arrangement. The origin of this inconsistency in the energetic hierarchy is not yet clear. It may be related either to the presence of some defects or disordering in the phases of interest, or the choice of exchange-correlation functional in the DFT calculations. In fact, the relative energy of $\alpha-\mathrm{Sb}_{2} \mathrm{O}_{3}$ became $0.004 \mathrm{eV} /$ f.u. lower than that of $\beta-\mathrm{Sb}_{2} \mathrm{O}_{3}$ when we used the Heyd-Scuseria-Ernzerhof (HSE06) ${ }^{35-37}$ hybrid functional for the same calculation. More detailed study is required in the future to discuss the detailed energetic hierarchy among polymorphs, which is beyond the scope of the present study.

Some of the dynamically stable structures found in the present study have not yet been revealed by experiments. They are $\langle 100\rangle$-LS and valentinite-LS for $\mathrm{As}_{2} \mathrm{O}_{3}$, claudetite-type for $\mathrm{Sb}_{2} \mathrm{O}_{3}$, and $\langle 111\rangle-\mathrm{LS}$, bixbyite-LS, and valentinite-LS

(a)

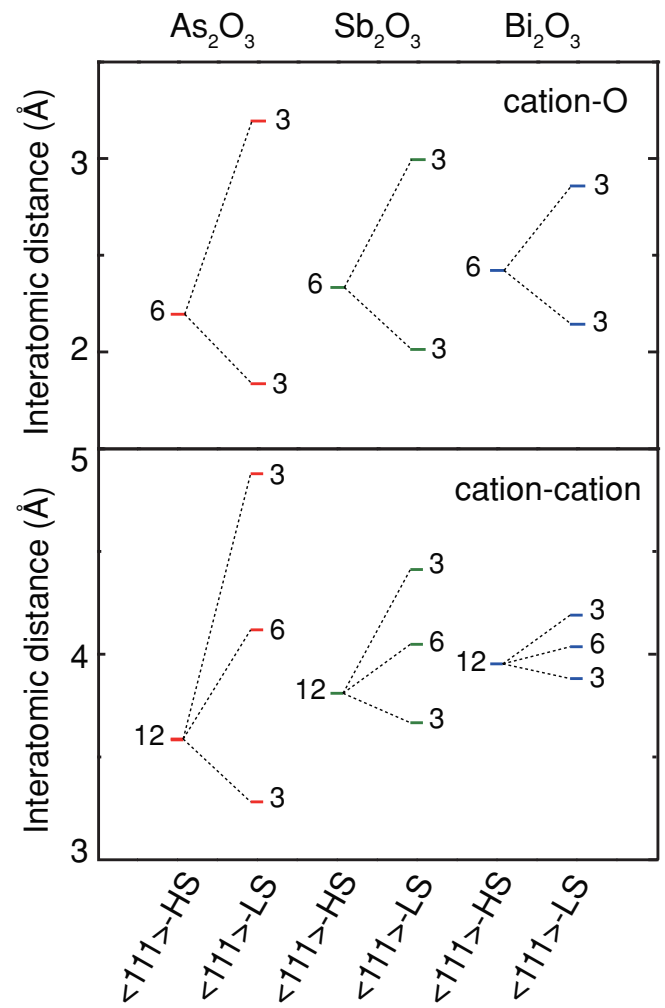

FIG. 5. (Color online) (a) Cation-O and (b) cation-cation interatomic distances in the $\langle 111\rangle$-HS and -LS arrangements of the three sesquioxides. The numbers beside the bars show the degeneracy. 

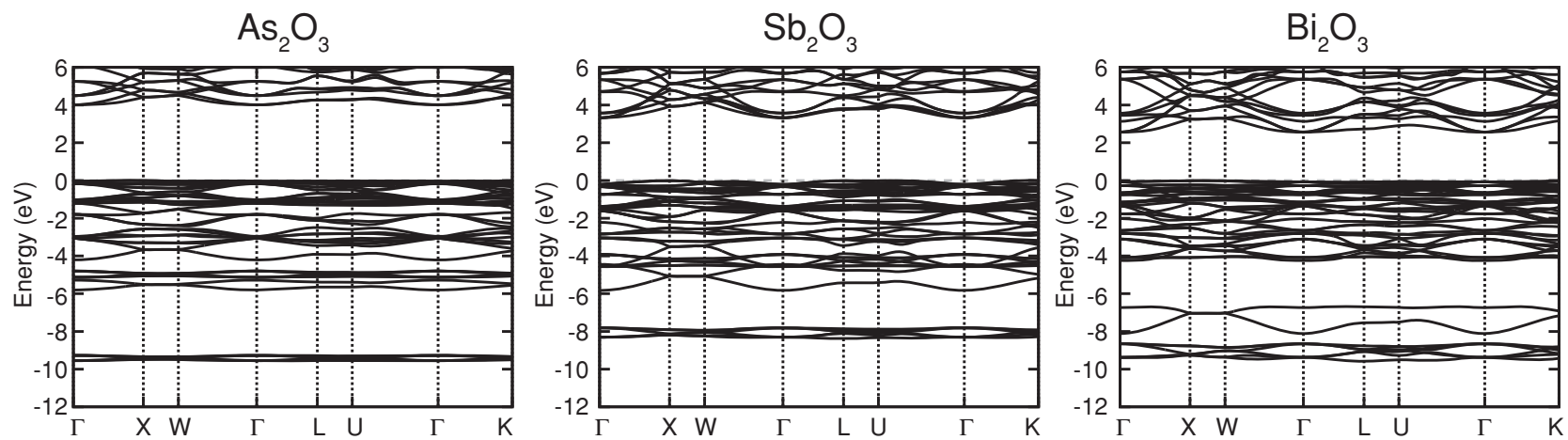

FIG. 6. Electronic band structures of the $\langle 111\rangle$-LS arrangements for the three sesquioxides. The top of the valence band is set to 0 on the vertical axis.

for $\mathrm{Bi}_{2} \mathrm{O}_{3}$. With the exception of $\langle 111\rangle-\mathrm{LS}$ for $\mathrm{Bi}_{2} \mathrm{O}_{3}$, their formation energies are within $0.1 \mathrm{eV} / \mathrm{f}$.u. of that for the structure with the lowest energy, implying the high probability of formation under controlled experimental conditions.

\section{B. Local atomic arrangements of dynamically stable structures}

As described in the previous section, the energy of each array model is lowered considerably upon symmetry breaking and local atomic distortion. In this section, the local atomic arrangements of the stable structures are investigated.

Among the five array models based on the defective fluorite structure, the $\langle 111\rangle$-LS arrangements of the three sesquioxides have the same space group. The $\langle 111\rangle$-HS arrangement has the $P n \overline{3} m$ space group with a 10 -atom unit cell. It transforms to the $\langle 111\rangle$-LS arrangement, which has the $F d \overline{3} m$ space group with an 80-atom unit cell, in the three sesquioxides. We therefore compare the HS and LS arrangements of the three sesquioxides with the $\langle 111\rangle$ array model in detail. Both HS and LS arrangements have one cation site and one O site. The cation is coordinated by six $\mathrm{O}$ ions and twelve cations. Figure 4 shows the $\langle 111\rangle$-HS and -LS arrangements of $\mathrm{As}_{2} \mathrm{O}_{3}$ from the [110] direction. As can be clearly seen in Fig. 4, ions tend to aggregate in the LS arrangement, while they are evenly distributed in the $\mathrm{HS}$ arrangement. Similar trends can be observed in $\mathrm{Sb}_{2} \mathrm{O}_{3}$ and $\mathrm{Bi}_{2} \mathrm{O}_{3}$. Figure 5 shows (a) cation-O and (b) cation-cation interatomic distances in the $\langle 111\rangle$-HS and -LS arrangements of the three sesquioxides. The numbers beside the bars show the degeneracy. All cation-O and cation-cation interatomic distances are the same in the HS arrangement. However, in the LS arrangement some of them are shortened and others are elongated by the local distortion. The volume also changes with the structural change. Table III shows the lattice constant and the volume expansion of the 80-atom cubic-cell in the $\langle 111\rangle$-HS and -LS arrangements for the three sesquioxides. The volume increases by $47 \%$ in $\mathrm{As}_{2} \mathrm{O}_{3}$, but only by $6 \%$ in $\mathrm{Bi}_{2} \mathrm{O}_{3}$. With increasing atomic aggregation and volume, the atomic density locally decreases in the $\langle 111\rangle$-LS arrangement. (a) $<111>-H S$
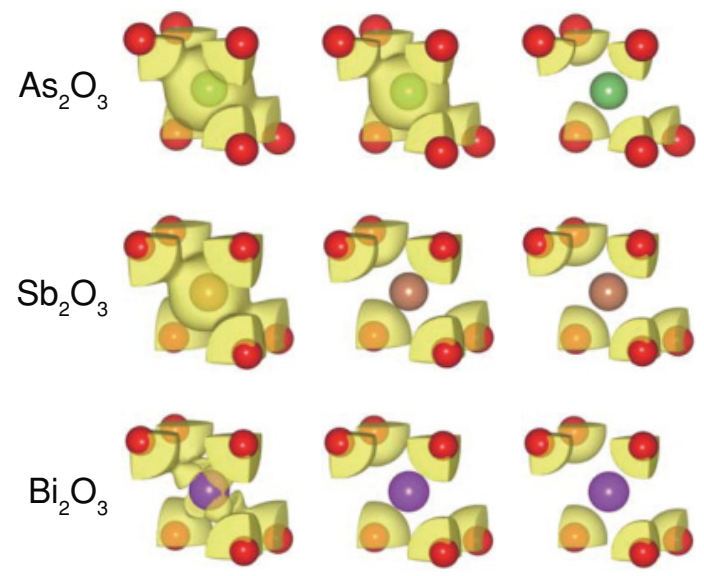

$$
\rho=0.3\left(\AA^{-3}\right)
$$

0.6

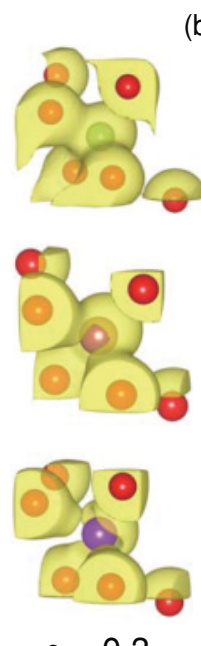

$\rho=0.3$ (b) $<111>-$ LS
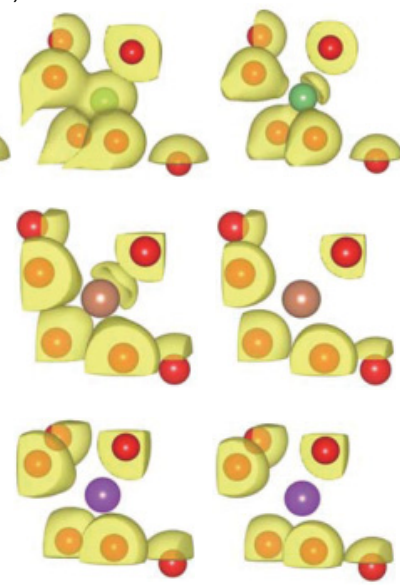

0.4

0.6

FIG. 7. (Color online) Isosurfaces of the valence electron density distribution around the cation in the (a) $\langle 111\rangle$-HS and (b) $\langle 111\rangle$-LS arrangements of the three sesquioxides. Part of the unit cell is extracted. Red spheres are oxygen ions. Green, brown, and violet spheres are cations. 
TABLE III. Lattice constant and volume expansion of 80-atom cubic-cell in the $\langle 111\rangle$-HS and -LS arrangements of the three sesquioxides.

\begin{tabular}{lccc}
\hline \hline & $\langle 111\rangle$-HS & $\langle 111\rangle$-LS & Volume expansion \\
\hline $\mathrm{As}_{2} \mathrm{O}_{3}$ & $10.13 \AA$ & $11.54 \AA$ & $47 \%$ \\
$\mathrm{Sb}_{2} \mathrm{O}_{3}$ & $10.77 \AA$ & $11.42 \AA$ & $19 \%$ \\
$\mathrm{Bi}_{2} \mathrm{O}_{3}$ & $11.18 \AA$ & $11.41 \AA$ & $6 \%$ \\
\hline \hline
\end{tabular}

\section{Electronic structures of dynamically stable structures}

$\mathrm{Sb}_{2} \mathrm{O}_{3}$ and $\mathrm{Bi}_{2} \mathrm{O}_{3}$ have been experimentally found to have clear band gaps. ${ }^{38-41}$ Although details of the measurement were not given, $\mathrm{As}_{2} \mathrm{O}_{3}$ has also been reported to have a band gap. $^{42}$ In spite of these reports, the HS structures with a 10 -atom unit cell, i.e., the $\langle 100\rangle$-HS, $\langle 110\rangle$-HS, and $\langle 111\rangle$-HS arrangements, for the three sesquioxides have either a metallic or semimetallic electronic structure in which an energy band crosses the Fermi level or the top of the valence band is higher than the bottom of the conduction band. When symmetry breaking and local distortion are introduced into these structures, band gaps appear. The electronic band structures of the $\langle 111\rangle$-LS arrangements for the three sesquioxides are shown in Fig. 6. All LS arrangements for the three sesquioxides have clear band gaps, which are summarized in Table IV. Since the LS arrangement could not be obtained in some of the array models, some values are missing. $\alpha-\mathrm{Sb}_{2} \mathrm{O}_{3}$ and $\beta-\mathrm{Sb}_{2} \mathrm{O}_{3}$ have been reported to have band gaps of $4 \mathrm{eV}$ (Refs. 38-40) and $3.3 \mathrm{eV},{ }^{38}$ respectively. The corresponding theoretical values in this work are $3.33 \mathrm{eV}(\langle 111\rangle-\mathrm{LS})$ and $2.20 \mathrm{eV}$ (valentinite-LS), respectively. The underestimation may be ascribed to the use of the GGA for the exchange-correlation functional. As a matter of fact, the band gaps similarly calculated with the HSE06 hybrid functional are $4.28 \mathrm{eV}(\langle 111\rangle-\mathrm{LS})$ and $3.60 \mathrm{eV}$ (valentinite-LS), respectively, which are much closer to the experimental results. The band structures by the HSE06 calculations are seemingly the same as those by the GGA except for the improved band gap.

We found a trend that the band gap decreases with the increase of the relative energy among the different arrangements. The local distortion affects the electronic structure significantly. The inclusion of symmetry breaking and local distortion is therefore essential for accurately describing the energetics and electronic structures of the three sesquioxides.

\section{Formation of a "lone pair"}

In the sesquioxides of late group-15 elements, two $n s$ electrons remain in the valence band in the formal sense. When they are localized to the cations and form an asymmetric charge distribution, they are referred to as a "lone pair". The formation

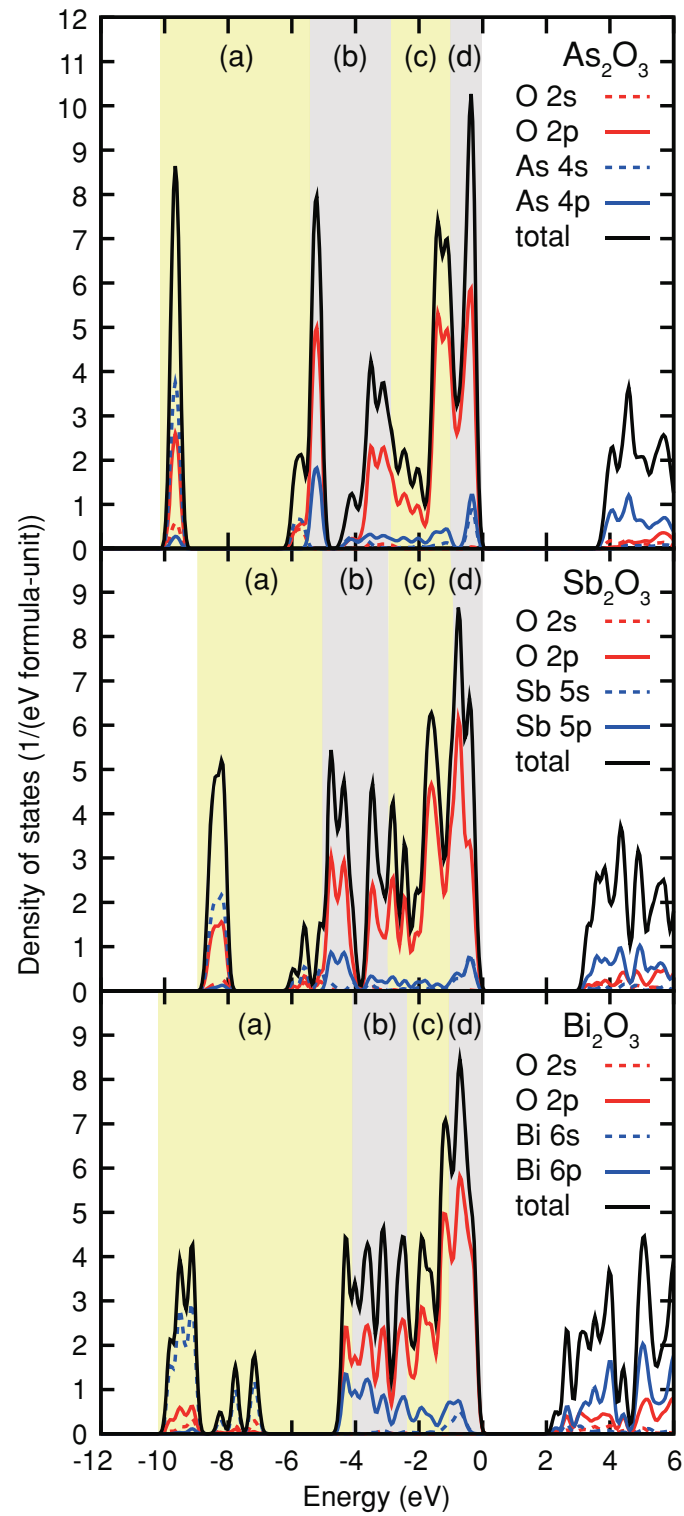

FIG. 8. (Color online) Partial electron density of states of the $\langle 111\rangle$-LS arrangement for the three sesquioxides. The top of the valence band is set to 0 .The black line represents the total density of states. Broken and solid red lines show the O $2 s$ and O $2 p$ states, respectively. Broken and solid blue lines represent the cation $n s$ and $n p$ states, respectively. Four regions, (a)-(d), include the same number of valence electrons.

of a lone pair often plays a central role in determining the local atomic arrangement. To examine the origin of the local atomic distortion and symmetry breaking, we examine the charge distribution of the sesquioxides in detail.

TABLE IV. Calculated GGA band gaps of LS structures. Values in square brackets are HSE06 band gaps. The unit is an electronvolt.

\begin{tabular}{|c|c|c|c|c|c|}
\hline & $\langle 100\rangle-\mathrm{LS}$ & $\langle 111\rangle-\mathrm{LS}$ & bixbyite-LS & valentinite-LS & $P 2_{1} / c$ \\
\hline $\mathrm{As}_{2} \mathrm{O}_{3}$ & 3.64 & $4.01[5.34]$ & & 3.23 [3.92] & 3.58 \\
\hline $\mathrm{Sb}_{2} \mathrm{O}_{3}$ & & $3.33[4.28]$ & & $2.20[3.60]$ & 2.51 \\
\hline $\mathrm{Bi}_{2} \mathrm{O}_{3}$ & 1.61 [2.73] & 2.57 [3.93] & 2.58 & 1.74 [2.89] & 2.29 \\
\hline
\end{tabular}


(a)
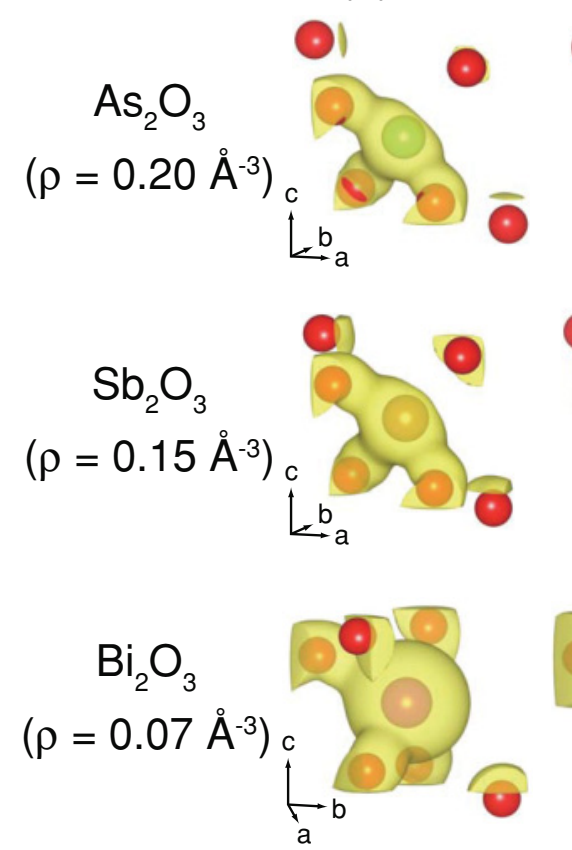

(b)
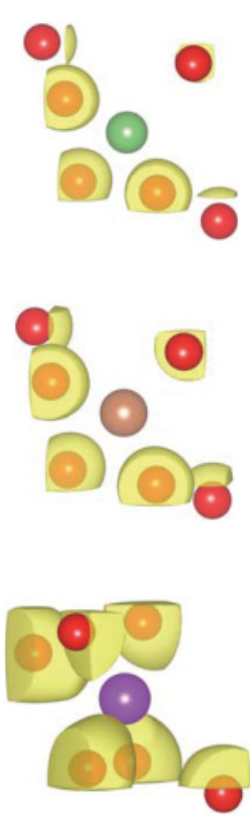

(c)
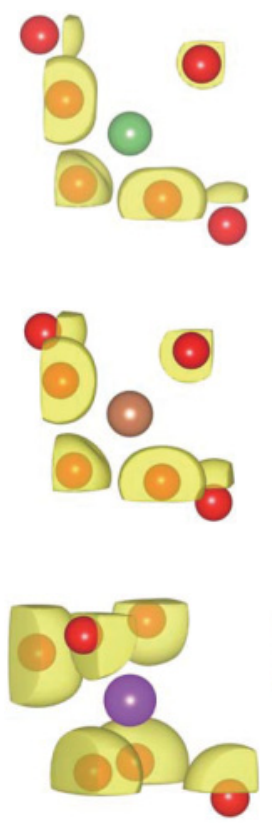

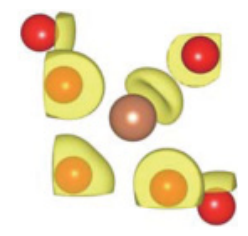

(d)
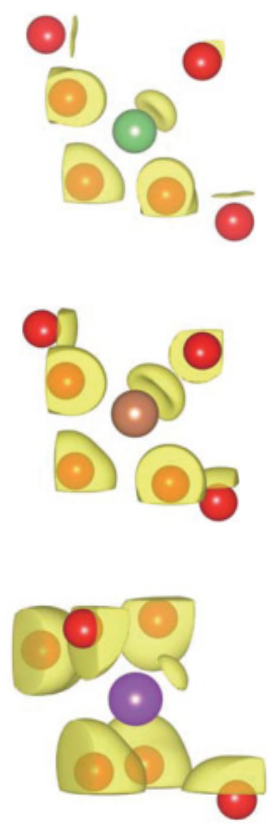

FIG. 9. (Color online) Isosurface of the valence electron density distribution in each region (see Fig. 8) around the cation in the $\langle 111\rangle$-LS arrangement of the three sesquioxides. The isosurfaces of $0.20,0.15$, and $0.07 \AA^{-3}$ are shown for $\mathrm{As}_{2} \mathrm{O}_{3}, \mathrm{Sb}_{2} \mathrm{O}_{3}$, and $\mathrm{Bi}_{2} \mathrm{O}_{3}$, respectively. $\mathrm{Red}$ spheres are oxygen ions. Green, brown, and violet spheres are cations.

Figure 7 shows the isosurfaces of the valence electron density distribution around the cation in the $\langle 111\rangle$-HS and -LS arrangements of the three sesquioxides. The atomic arrangements can be better visualized in the HS arrangement. The cation is coordinated by six $\mathrm{O}$ ions and two empty sites. The empty sites are located at the front-bottom-left and top-right-back in the cubic structure shown in Fig. 7(a). In the transformation from HS to LS, O ions move toward the empty site at the front-bottom-left as can be seen in Fig. 7(b). Then the other empty site at the top-right-back has a larger open space than the corresponding space in the HS arrangement. In the HS arrangement, electrons are spherically distributed around the cation, as shown in Fig. 7(a). The isosurfaces of $\geqslant 0.6 \AA^{-3}$ for $\mathrm{As}_{2} \mathrm{O}_{3}, \geqslant 0.4 \AA^{-3}$ for $\mathrm{Sb}_{2} \mathrm{O}_{3}$, and $\geqslant 0.3 \AA^{-3}$ for $\mathrm{Bi}_{2} \mathrm{O}_{3}$ cannot be seen around the cation. On the other hand, as shown in Fig. 7(b), the charge density around the cation became asymmetric and more localized in the LS arrangements. The charge distribution along the direction of the empty site at the top-back-right can be clearly seen in the isosurfaces of $0.6 \AA^{-3}$ for $\mathrm{As}_{2} \mathrm{O}_{3}, 0.4 \AA^{-3}$ for $\mathrm{Sb}_{2} \mathrm{O}_{3}$, and $0.3 \AA^{-3}$ for $\mathrm{Bi}_{2} \mathrm{O}_{3}$.

To inspect the formation of the lone pair in more detail, the partial charge-density distribution is investigated. Figure 8 shows the electron density of states in the $\langle 111\rangle$-LS arrangement for the three sesquioxides. The top of the valence band is set to zero. The valence band is mainly composed of the $\mathrm{O}$ $2 p$ state with minor contributions of the cation $n s$ and $n p$ states ( $n=4,5$, and 6 for As, $\mathrm{Sb}$, and Bi, respectively). When the fully filled $(n-1) d$ electrons of the cations are excluded, the three sesquioxides have $2 \times 2+3 \times 6=22$ valence electrons in the $\mathrm{O} 2 p$ band per formula unit of $M_{2} \mathrm{O}_{3}$. The $\mathrm{O} 2 p$ band is divided into four regions, (a)-(d), to include the same number of electrons, 22/4, in each region as shown in Fig. 8.
The highest-energy region, (d), is mainly composed of $\mathrm{O} 2 p$ states forming an antibonding interaction with the cation $n s p$ state. The isosurface of the partial valence electron density distribution in each region is shown in Fig. 9. The isosurfaces of $0.20,0.15$, and $0.07 \AA^{-3}$ are shown for $\mathrm{As}_{2} \mathrm{O}_{3}, \mathrm{Sb}_{2} \mathrm{O}_{3}$, and $\mathrm{Bi}_{2} \mathrm{O}_{3}$, respectively. The model of $\mathrm{Bi}_{2} \mathrm{O}_{3}$ in Fig. 9 is slightly tilted from the other two models for easy visualization of the electron localization. As shown in Fig. 9, only the isosurface of region (d) is elongated toward the direction of the empty site in all three sesquioxides. This indicates that the cation $n s$ and $n p$ electrons in region (d) contribute significantly to the anisotropic and localized charge distribution, which is consistent with the idea of a lone pair on the cation. The magnitudes of the anisotropy and localization become less significant in the order $\mathrm{As}_{2} \mathrm{O}_{3}, \mathrm{Sb}_{2} \mathrm{O}_{3}$, and $\mathrm{Bi}_{2} \mathrm{O}_{3}$. This is the same trend as the lattice distortion associated with the $\langle 111\rangle$-HS to -LS transformation. This means that the formation of the lone pair is most significant in $\mathrm{As}_{2} \mathrm{O}_{3}$. The formation of the lone pair is thus confirmed to play a central role in the local atomic distortion and symmetry breaking.

\section{SUMMARY}

First-principles lattice-dynamics calculations were systematically performed for $M_{2} \mathrm{O}_{3}(M=\mathrm{As}, \mathrm{Sb}, \mathrm{Bi})$. Dynamically stable LS structures were searched for, including atomic displacements along imaginary modes of lattice vibrations that appear in HS defective fluorite structures. The atomic arrangements, electronic structures, and energetics of the LS structures were then examined in detail. The major results in this study can be summarized as follows: 
(i) All five array models with the HS defective fluorite arrangements were dynamically unstable against various vibration modes. When symmetry breaking and local distortion were included, some structures became dynamically stable. The majority of the experimentally reported structures (arsenolite- $\mathrm{As}_{2} \mathrm{O}_{3}, \alpha-\mathrm{Sb}_{2} \mathrm{O}_{3}, \beta-\mathrm{Sb}_{2} \mathrm{O}_{3}$, and $\beta-\mathrm{Bi}_{2} \mathrm{O}_{3}$ ) were obtained by this procedure. At the same time, some dynamically stable structures not yet revealed by experiments were discovered. They are $\langle 100\rangle$-LS and valentinite-LS for $\mathrm{As}_{2} \mathrm{O}_{3}$, claudetite-type for $\mathrm{Sb}_{2} \mathrm{O}_{3}$, and $\langle 111\rangle$-LS, bixbyite-LS, and valentinite-LS for $\mathrm{Bi}_{2} \mathrm{O}_{3}$. With the exception of $\langle 111\rangle$-LS for $\mathrm{Bi}_{2} \mathrm{O}_{3}$, their formation energies were within $0.1 \mathrm{eV} /$ f.u. of that for the structure with the lowest energy, implying a high probability of formation under controlled process conditions (Fig. 3).

(ii) Ions tend to aggregate in the LS arrangement, whereas they are evenly distributed in the HS arrangement (Fig. 4). All cation-anion and cation-cation interatomic distances were the same in the HS arrangement. However, in the LS arrangement some of them were shortened and others were elongated by the local distortion (Fig. 5). With increasing atomic aggregation and volume, the atomic density locally decreases in the LS arrangement.

(iii) Although the HS arrangements for the three sesquioxides exhibited either a metallic or semimetallic electronic structure, all LS arrangements exhibited clear band gaps
(Table IV), in good agreement with experimental reports. The inclusion of symmetry breaking and local distortion is therefore essential for accurately describing the energetics and electronic structures of the three sesquioxides.

(iv) In the HS arrangements, electrons are spherically distributed around the cation. On the other hand, in the LS arrangements, the charge density around the cation became asymmetric and elongated along the direction of the empty site (Fig. 7). The cation $n s$ and $n p$ ( $n=4,5$, and 6 for As, $\mathrm{Sb}$, and $\mathrm{Bi}$, respectively) electrons near the top of the valence band contribute significantly to the anisotropic and localized charge distribution (Fig. 9), i.e., the formation of the lone pair. This became less significant in the order $\mathrm{As}_{2} \mathrm{O}_{3}, \mathrm{Sb}_{2} \mathrm{O}_{3}$, and $\mathrm{Bi}_{2} \mathrm{O}_{3}$, which was the same trend as the lattice distortion. The formation of the lone pair was thus confirmed to play a central role in the local atomic distortion and symmetry breaking.

\section{ACKNOWLEDGMENTS}

This study was supported by both a Grant-in-Aid for Scientific Research (A) and a Grant-in-Aid for Scientific Research on Priority Areas "Nano Materials Science for Atomic Scale Modification 474" from the Ministry of Education, Culture, Sports, Science and Technology (MEXT) of Japan. A.M. thanks the Japan Society for the Promotion of Science. *tanaka@cms.mtl.kyoto-u.ac.jp

${ }^{1}$ F. Pertlik, Czech. J. Phys. B 28, 170 (1978).

${ }^{2}$ A. J. Frueh, Am. Mineral. 36, 833 (1951).

${ }^{3}$ F. Pertlik, Monatsh. Chem. 109, 277 (1978).

${ }^{4}$ D. Ležal and K. Koňak, J. Non-Cryst. Solids 192\&193, 187 (1995).

${ }^{5}$ C. Svensson, Acta. Crystallogr., Sect. B 30, 458 (1974).

${ }^{6}$ C. Svensson, Acta. Crystallogr., Sect. B 31, 2016 (1975).

${ }^{7}$ S. J. Gilliam, J. O. Jensen, A. Banerjee, D. Zeroka, S. J. Kirkby, and C. N. Merrowa, Spectrochim. Acta Part A 60, 425 (2004).

${ }^{8}$ R. G. Orman and D. Holland, J. Solid State Chem. 180, 2587 (2007).

${ }^{9}$ E. I. Voit, A. E. Panasenko, and L. A. Zemnukhova, J. Struct. Chem. 50, 60 (2009).

${ }^{10}$ M. Drache, P. Roussel, and J. Wignacourt, Chem. Rev. 107, 80 (2007).

${ }^{11}$ N. M. Sammes, G. A. Tompsett, H. Näfe, and F. Aldinger, J. Eur. Ceram. Soc. 19, 1801 (1999).

${ }^{12}$ L. G. Sillen, Ark. Kemi 12A, 1 (1937).

${ }^{13}$ V. G. Gattow and H. Schröder, Z. Anorg. Allg. Chem. 318, 176 (1962).

${ }^{14}$ V. G. Gattow and D. Schutze, Z. Anorg. Allg. Chem. 328, 44 (1964).

${ }^{15}$ E. M. Levin and R. S. Roth, J. Res. Natl. Bur. Stand. 68A, 197 (1964).

${ }^{16}$ H. A. Harwig and A. G. Gerards, Thermochim. Acta 28, 121 (1979).

${ }^{17}$ C. N. R. Rao, G. V. S. Rao, and S. Ramdas, J. Phys. Chem. 73, 672 (1969).
${ }^{18}$ N. Cornei, N. Tancret, F. Abraham, and O. Mentré, Inorg. Chem. Commun. 45, 4886 (2006).

${ }^{19}$ A. F. Gualtieri, S. Imovilli, and M. Prudenziati, Powder Diff. 12, 90 (1997).

${ }^{20}$ S. K. Blower and C. Greaves, Acta Crystallogr. C 44, 587 (1988).

${ }^{21}$ T. Takahashi, H. Iwahara, and Y. Nagai, J. Appl. Electrochem. 2, 97 (1972)

${ }^{22}$ A. Matsumoto, Y. Koyama, and I. Tanaka, Phys. Rev. B 81, 094117 (2010).

${ }^{23}$ F. Pertlik, Monatsh. Chem. 106, 755 (1975).

${ }^{24}$ H. A. Harwig, Z. Anorg. Allg. Chem. 444, 151 (1978).

${ }^{25}$ P. E. Blöchl, Phys. Rev. B 50, 17953 (1994).

${ }^{26}$ G. Kresse and J. Hafner, Phys. Rev. B 47, R558 (1993).

${ }^{27}$ G. Kresse and J. Furthmüller, Phys. Rev. B 54, 11169 (1996).

${ }^{28}$ G. Kresse and J. Furthmüller, Comput. Mater. Sci. 6, 15 (1996).

${ }^{29}$ J. P. Perdew, K. Burke, and M. Ernzerhof, Phys. Rev. Lett. 77, 3865 (1996).

${ }^{30}$ H. J. Monkhorst and J. D. Pack, Phys. Rev. B 13, 5188 (1976).

${ }^{31} \mathrm{~A}$. Togo, [http://fropho.sourceforge.net/].

${ }^{32}$ A. Togo, F. Oba, and I. Tanaka, Phys. Rev. B 77, 184101 (2008).

${ }^{33}$ A. Togo, F. Oba, and I. Tanaka, Phys. Rev. B 78, 134106 (2008).

${ }^{34}$ K. A. Becker, K. Plieth, and I. N. Stranski, Z. Anorg. Allg. Chem. 266, 293 (1951).

${ }^{35}$ J. Heyd, G. E. Scuseria, and M. Ernzerhof, J. Chem. Phys. 118, 8207 (2003).

${ }^{36}$ A. V. Krukau, O. A. Vydrov, A. F. Izmaylov, and G. E. Scuseria, J. Chem. Phys. 125, 224106 (2006). 
${ }^{37}$ J. Heyd, G. E. Scuseria, and M. Ernzerhof, J. Chem. Phys. 124, 219906 (2006).

${ }^{38}$ C. Wood, B. V. Pelt, and A. Dwight, Phys. Status Solidi B 54, 701 (1972).

${ }^{39}$ N. Tigau, V. Ciupina, and G. Prodan, J. Cryst. Growth 277, 529 (2005).
${ }^{40}$ N. Tigau, V. Ciupina, and G. Prodan, J. Optoelectron. Adv. Mater. 8, 37 (2006).

${ }^{41}$ H. T. Fan, S. S. Pan, X. M. Teng, C. Ye, and G. H. Li, J. Phys. D 39, 1939 (2006).

${ }^{42}$ J. Portier, G. Campet, C. W. Kwon, J. Etourneau, and M. A. Subramanian, J. Int. J. Inorg. Mater. 3, 1091 (2001). 